Acta Crystallographica Section E

Structure Reports

Online

ISSN 1600-5368

\title{
Poly[octaaquadi- $\mu$-phosphato-tri-
} nickel(II)]. Corrigendum

\author{
Shouwen Jin, ${ }^{a *}$ Daqi Wang, ${ }^{b}$ Xinjun Gao, ${ }^{a}$ Xianhong Wen ${ }^{a}$ \\ and Jianzhong Zhou ${ }^{\text {a }}$ \\ ${ }^{\text {a}}$ Faculty of Science, ZheJiang Forestry University, Lin'An 311300, People's Republic \\ of China, and ${ }^{\mathbf{b}}$ Department of Chemistry, Liaocheng University, Shandong 252059, \\ People's Republic of China \\ Correspondence e-mail: jinsw@zjfc.edu.cn
}

Received 7 January 2008; accepted 21 January 2008

A correction is made to the name of the first author in Shouwen, Wang, Gao, Wen \& Zhou [Acta Cryst. (2008), E64, $\mathrm{m} 259]$.

In the paper by Shouwen, Wang, Gao, Wen \& Zhou [Acta Cryst. (2008), E64, m259], the name of the first author is given incorrectly. The correct name should be Shouwen Jin, as given above. 
Acta Crystallographica Section E

\section{Structure Reports}

Online

ISSN 1600-5368

\section{Poly[octaaquadi- $\mu$-phosphato-tri- nickel(II)]}

\section{Jin Shouwen, ${ }^{\text {a* Daqi Wang, }}{ }^{\mathrm{b}}$ Xinjun Gao, ${ }^{\mathrm{a}}$ Xianhong Wen ${ }^{\mathrm{a}}$ and Jianzhong Zhou ${ }^{\mathrm{a}}$}

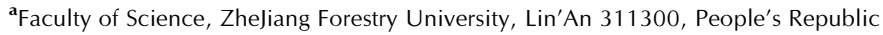
of China, and 'bepartment of Chemistry, Liaocheng University, Shandong 252059, People's Republic of China

Correspondence e-mail: jinsw@zjfc.edu.cn

Received 26 October 2007; accepted 14 December 2007

Key indicators: single-crystal X-ray study; $T=298 \mathrm{~K}$; mean $\sigma(\mathrm{P}-\mathrm{O})=0.003 \AA$; $R$ factor $=0.030 ; w R$ factor $=0.080 ;$ data-to-parameter ratio $=10.1$.

In the title compound, $\left[\mathrm{Ni}_{3}\left(\mathrm{PO}_{4}\right)_{2}\left(\mathrm{H}_{2} \mathrm{O}\right)_{8}\right]_{n}$, which was synthesized hydrothermally, all the $\mathrm{Ni}$ atoms are located in slightly distorted octahedral coordination environments. Two phosphate groups and two $\mathrm{Ni}$ atoms share a centrosymmetric four-membered ring and an eight-membered ring such that the four-membered ring is inside the eight-membered ring. The eight-membered rings are connected with the other $\mathrm{Ni}$ atoms (lying on centres of symmetry) through phosphate anions, generating a one-dimensional chain structure. Adjacent chains are connected through hydrogen bonds, forming a threedimensional network.

\section{Related literature}

For related literature, see: Chang et al. (2004); Gao et al. (1999); Ke et al. (2001); Kuratieva et al. (2003); Nardelli (1999); Sanz et al. (1999); Wang \& Gao (2005a,b).

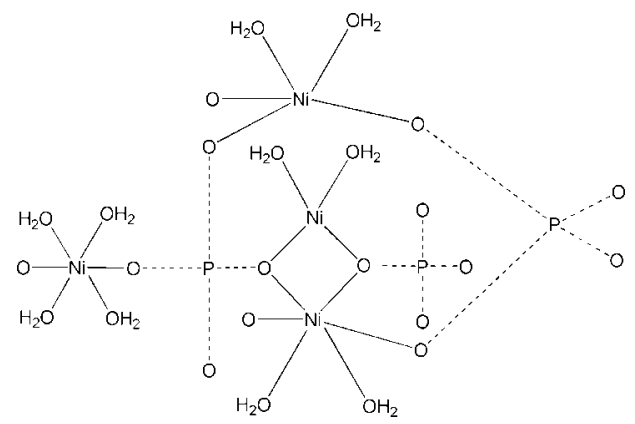

\section{Experimental}

Crystal data

$\begin{array}{ll}{\left[\mathrm{Ni}_{3}\left(\mathrm{PO}_{4}\right)_{2}\left(\mathrm{H}_{2} \mathrm{O}\right)_{8}\right]} & \text { Monoclinic, } C 2 / m \\ M_{r}=510.14 & a=9.963(3) \AA\end{array}$

$$
\begin{aligned}
& b=13.225(4) \AA \\
& c=4.6406(14) \AA \\
& \beta=104.730(3)^{\circ} \\
& V=591.4(3) \AA^{3} \\
& Z=2
\end{aligned}
$$

Data collection

Bruker SMART APEX CCD Diffractometer

Absorption correction: multi-scan (SADABS; Sheldrick, 1996)

$T_{\text {min }}=0.351, T_{\max }=0.414$ (expected range $=0.291-0.343$ )

\section{Refinement}

$R\left[F^{2}>2 \sigma\left(F^{2}\right)\right]=0.030$

$w R\left(F^{2}\right)=0.080$

$S=1.10$

553 reflections
Mo $K \alpha$ radiation

$\mu=5.09 \mathrm{~mm}^{-1}$

$T=298$ (2) K

$0.26 \times 0.23 \times 0.21 \mathrm{~mm}$

1516 measured reflections 553 independent reflections

\begin{tabular}{|c|c|c|c|c|}
\hline$D-\mathrm{H} \cdots A$ & $D-\mathrm{H}$ & $\mathrm{H} \cdots A$ & $D \cdots A$ & $D-\mathrm{H} \cdots A$ \\
\hline $\mathrm{O} 4-\mathrm{H} 1 \cdots \mathrm{O} 1^{\mathrm{i}}$ & 0.85 & 1.87 & $2.700(3)$ & 164 \\
\hline $\mathrm{O} 4-\mathrm{H} 2 \cdots \mathrm{O}^{\mathrm{ii}}$ & 0.85 & 1.98 & $2.731(3)$ & 147 \\
\hline $\mathrm{O} 5-\mathrm{H} 3 \cdots \mathrm{O}^{\mathrm{iii}}$ & 0.85 & 2.00 & $2.761(3)$ & 149 \\
\hline $\mathrm{O} 5-\mathrm{H} 4 \cdots \mathrm{O} 4^{\mathrm{iv}}$ & 0.85 & 2.07 & $2.906(3)$ & 167 \\
\hline
\end{tabular}
513 reflections with $I>2 \sigma(I)$ $R_{\text {int }}=0.046$
Table 1

Hydrogen-bond geometry $\left(\AA{ }^{\circ}\right)$.

Symmetry codes: (i) $-x+2,-y,-z+2$; $\quad$ (ii) $\quad-x+2,-y,-z+3$; $-x+1,-y,-z+1$; (iv) $x-\frac{1}{2},-y+\frac{1}{2}, z-1$.

Data collection: SMART (Bruker, 1997); cell refinement: SMART; data reduction: SAINT (Bruker, 1997); program(s) used to solve structure: SHELXS97 (Sheldrick, 1997a); program(s) used to refine structure: SHELXL97 (Sheldrick, 1997a); molecular graphics: SHELXTL (Sheldrick, 1997b); software used to prepare material for publication: SHELXTL.

The authors thank the Zhejiang Forestry University Science Foundation for financial support.

Supplementary data and figures for this paper are available from the IUCr electronic archives (Reference: CS2061).

\section{References}

Bruker (1997). SAINT and SMART. Bruker AXS Inc., Madison, Wisconsin, USA.

Chang, J., Hwang, J., Jhung, S. H., Park, S. E., Ferey, G. \& Cheetham, A. K. (2004). Angew. Chem. Int. Ed. 43, 2819-2822.

Gao, Q., Guillou, N., Nogues, M., Cheetham, A. K. \& Ferey, G. (1999). Chem. Mater. 11, 2937-2947.

Ke, Y. X., He, G. F., Li, J. M., Zhang, Y. G. \& Lu, S. M. (2001). New J. Chem. 25 , 1627-1630.

Kuratieva, N. V., Naumova, M. I., Naumov, D. Yu. \& Podberezskaya, N. V. (2003). Acta Cryst. C59, i1-i3.

Nardelli, M. (1999). J. Appl. Cryst. 32, 563-571.

Sanz, F., Parada, C., Rojo, J. M. \& Ruiz, V. C. (1999). Chem. Mater. 11, $2673-$ 2679.

Sheldrick, G. M. (1996). SADABS. University of Göttingen, Germany.

Sheldrick, G. M. (1997a). SHELXS97 and SHELXL97. University of Göttingen, Germany.

Sheldrick, G. M. (1997b). SHELXTL. Version 5.10. Bruker AXS Inc., Madison Wisconsin, USA.

Wang, X. L. \& Gao, Q. M. (2005a). J. Inorg. Mater. 20, 699-705.

Wang, X. L. \& Gao, Q. M. (2005b). Chin. J. Inorg. Chem. 19, 73-76. 


\section{supporting information}

Acta Cryst. (2008). E64, m259 [https://doi.org/10.1107/S1600536807067050]

Poly[octaaquadi- $\mu$-phosphato-trinickel(II)]

\section{Jin Shouwen, Daqi Wang, Xinjun Gao, Xianhong Wen and Jianzhong Zhou}

\section{S1. Comment}

In recent years, nickel phosphates as a rich class of inorganic materials, have undergone significant expansion due to their potential application (Chang et al., 2004; Gao et al., 1999; Sanz et al., 1999).

In general, current work has centered on their composite properties and the possibility of tuning their chemistry, by using effects from a wide variety of templates and of additives (Wang \& Gao, 2005a; Wang \& Gao, 2005b). However, investigations of new synthetic methods are still comparatively less exploited. For our interest in studying inorganic synthesis, herein we report the hydrothermal synthesis, and the crystal structure of the title compound. which is insoluble in water and in common organic solvents. It crystallizes in the monoclinic space group $\mathrm{C} 2 / \mathrm{m}$. The molecular structure, shown in Scheme 1, consists of three nickel ions, two phosphate ions, and eight water molecules. $\mathrm{The} \mathrm{PO}_{4}$ anions and the Ni cations are of normal valences, i.e. their valences are -3 , and +2 respectively.

The structure of nickel(II) phosphate octahydrate is shown in Fig. 1.

Both $\mathrm{Ni}$ atoms have a slightly distorted octahedral geometry. $\mathrm{Ni}$ atoms are located in two different environments, one $\mathrm{Ni}$ atom is surrounded by four water molecules and the remaining coordination sites are ocuppied by two oxygen atoms of two phosphates respectively. In this case, the $\mathrm{Ni}$ atoms are surrounded by two phosphates. The other $\mathrm{Ni}$ atom and its symmetry generated mate are surrounded by two water molecules, respectively. Oxygen atoms of the phosphates occupy the remaining four sites to complete the octahedral coordination. In this condition the two Ni atoms are each surrounded by three phosphates, such that two phosphates are parallel, and antiparallel with the other phosphate.

The $\mathrm{P}$ atoms of the phosphates do not participate in forming coordination bonds. All the $\mathrm{P}$ atoms make four $\mathrm{P}-\mathrm{O}$ bonds. $\mathrm{Ni}-\mathrm{O}$ bond distances are normal. The $\mathrm{P}-\mathrm{O}$ bond distances are in the range of 1.539 (3)-1.562 (3) $\AA$, the average value is $1.551 \AA$, and the $\mathrm{O}-\mathrm{P}-\mathrm{O}$ angles are in the range of 106.73 (16)-113.64 (16) degree, the average value is 110.18 degree. These geometry parameters are in good agreement with the reported results (Ke et al., 2001). The $\mathrm{Ni}-\mathrm{O}$ (water) bond lengths are within the reported range (Kuratieva et al., 2003).

The phosphate anion coordinates to two metal atoms in a chelating form with two oxygen atoms. A further bridge is to the third metal ion with one of the remaining oxygen atoms.

Two phosphate and two $\mathrm{Ni}$ atoms share a four-membered ring, and an eight-membered ring such that the four membered ring is inside the eight membered ring. In the four membered rings, the $\mathrm{Ni}-\mathrm{Ni}$ distance is $2.909 \AA$, while the distance between the Ni forming the four-membered rings and the Ni adjacent to the four-membered rings is $8.059 \AA$. The eight membered rings are connected with third symmetry-generated nickel atom linked through phosphate anions to provide an one dimensional chain structure. Adjacent chains were connected through hydrogen bonds to provide threedimensional network topology, which is shown in Fig. 2. 


\section{S2. Experimental}

All reagents and solvents were used as received.

Blue block crystals of the title compound were synthesized hydrothermally in a $23 \mathrm{ml}$ Teflon-lined autoclave by heating a mixture of 1-(4-(1H-imidazol -1-yl)butyl)-1H-imidazole ( $0.143 \mathrm{~g}, 0.75 \mathrm{mmol})$, nickel acetate dihydrate $(0.25 \mathrm{~g}, 1$ mmol), phosphoric acid $(0.11 \mathrm{~g}, 1 \mathrm{mmol})$, and deionized water $(6 \mathrm{ml})$ at 130 degree for 10 days. Then it was slowly cooled to room temperature, giving blue block crystals. Yield (based on $\mathrm{Ni}(\mathrm{Ac}) 2.2 \mathrm{H} 2 \mathrm{O}$ ): $0.11 \mathrm{~g}, 64.7 \%$.

\section{S3. Refinement}

Hydrogen atoms bound to water molecules were located in the Fourier difference map, and their distances were fixed, and subject to an $\mathrm{O}-\mathrm{H}=0.85 \AA$ with deviation of positive and negative $0.01 \AA$ restraint. The coordinates of the water $\mathrm{H}$ atoms were localized by applying the HYDROGEN program (Nardelli, 1999).

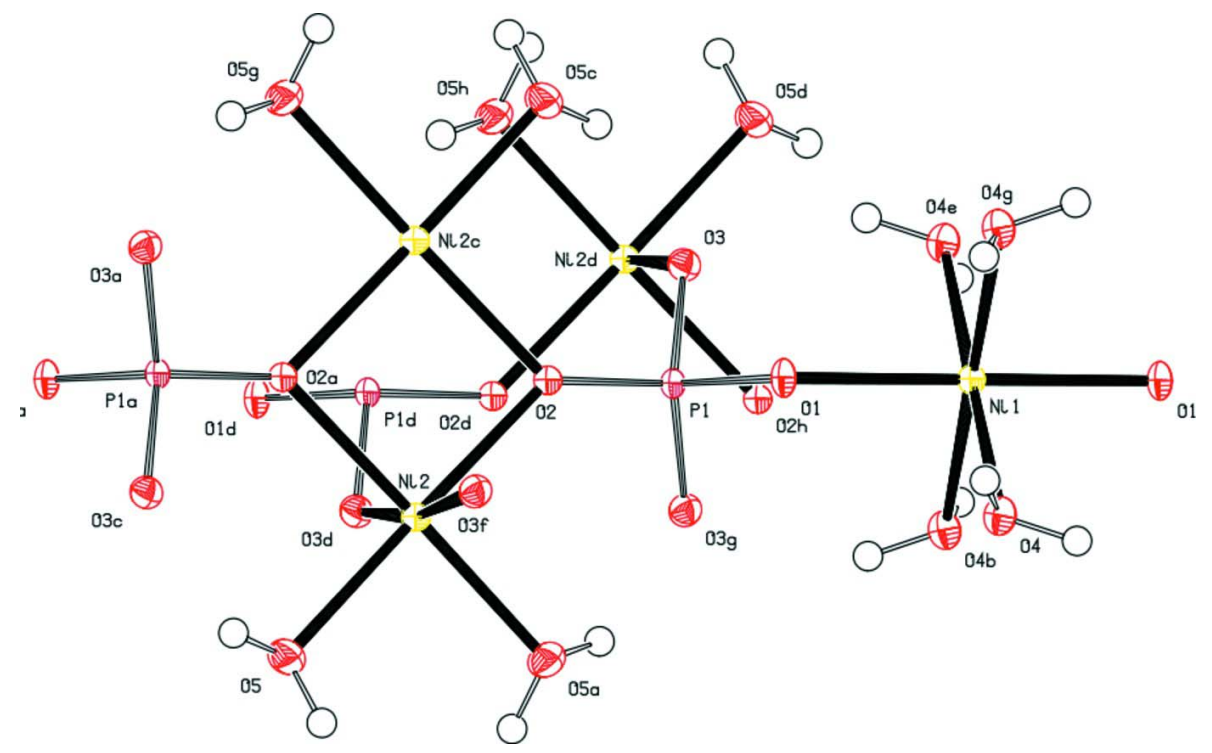

\section{Figure 1}

The structure of the title compound, showing the atom-numbering scheme. Displacement ellipsoids are drawn at the $50 \%$ probability level. The symmetry codes are: $(a)-x+1, y,-z+1 ;(b)-x+2, y,-z+3 ;(c)-x+1,-y,-z+1 ;(d) 1-x,-y, 2-z$; (e) $-x+2,-y,-z+3 ;$ (f) $x,-y,-1+z ;(g) x,-y, z ;(h) x,-y, 1+z$ 


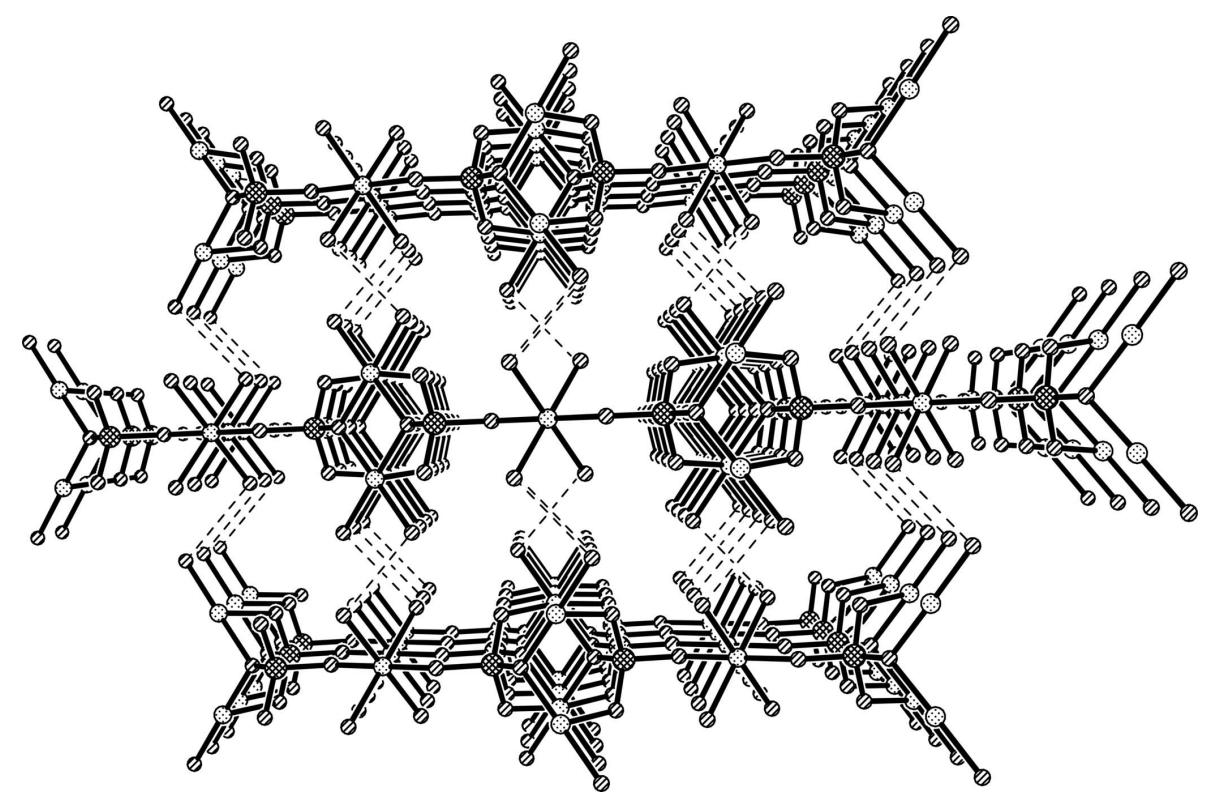

Figure 2

Three dimensional network structure formed via hydrogen bonds.

Poly[octaaquadi- $\mu$-phosphato-trinickel(II)]

Crystal data

$\left[\mathrm{Ni}_{3}\left(\mathrm{PO}_{4}\right)_{2}\left(\mathrm{H}_{2} \mathrm{O}\right)_{8}\right]$

$M_{r}=510.14$

Monoclinic, $C 2 / m$

$a=9.963(3) \AA$

$b=13.225(4) \AA$

$c=4.6406(14) \AA$

$\beta=104.730(3)^{\circ}$

$V=591.4(3) \AA^{3}$

Data collection

Bruker SMART APEX CCD Diffractometer

Radiation source: fine-focus sealed tube

Graphite monochromator

phi and $\omega$ scans

Absorption correction: multi-scan

(SADABS; Sheldrick, 1996)

$T_{\text {min }}=0.351, T_{\max }=0.414$

1516 measured reflections

Refinement

Refinement on $F^{2}$

Least-squares matrix: full

$R\left[F^{2}>2 \sigma\left(F^{2}\right)\right]=0.030$

$w R\left(F^{2}\right)=0.080$

$S=1.10$

553 reflections

55 parameters

0 restraints
$Z=2$

$F(000)=516$

$D_{\mathrm{x}}=2.865 \mathrm{Mg} \mathrm{m}^{-3}$

Mo $K \alpha$ radiation, $\lambda=0.71073 \AA$

$\mu=5.09 \mathrm{~mm}^{-1}$

$T=298 \mathrm{~K}$

Block, blue

$0.26 \times 0.23 \times 0.21 \mathrm{~mm}$

553 independent reflections

513 reflections with $I>2 \sigma(I)$

$R_{\text {int }}=0.046$

$\theta_{\max }=25.1^{\circ}, \theta_{\min }=2.6^{\circ}$

$h=-7 \rightarrow 11$

$k=-15 \rightarrow 15$

$l=-5 \rightarrow 5$

Primary atom site location: structure-invariant direct methods

Secondary atom site location: difference Fourier map

Hydrogen site location: inferred from neighbouring sites

$\mathrm{H}$-atom parameters constrained 
$w=1 /\left[\sigma^{2}\left(F_{\mathrm{o}}^{2}\right)+(0.0471 P)^{2}\right]$

where $P=\left(F_{\mathrm{o}}^{2}+2 F_{\mathrm{c}}^{2}\right) / 3$

$(\Delta / \sigma)_{\max }<0.001$

$$
\Delta \rho_{\max }=0.70 \mathrm{e} \AA^{-3}
$$$$
\Delta \rho_{\min }=-0.58 \text { e } \AA^{-3}
$$

\section{Special details}

Geometry. All e.s.d.'s (except the e.s.d. in the dihedral angle between two 1.s. planes) are estimated using the full covariance matrix. The cell e.s.d.'s are taken into account individually in the estimation of e.s.d.'s in distances, angles and torsion angles; correlations between e.s.d.'s in cell parameters are only used when they are defined by crystal symmetry. An approximate (isotropic) treatment of cell e.s.d.'s is used for estimating e.s.d.'s involving 1.s. planes.

Refinement. Refinement of $F^{2}$ against ALL reflections. The weighted $R$-factor $w R$ and goodness of fit $S$ are based on $F^{2}$, conventional $R$-factors $R$ are based on $F$, with $F$ set to zero for negative $F^{2}$. The threshold expression of $F^{2}>\sigma\left(F^{2}\right)$ is used only for calculating $R$-factors (gt) etc. and is not relevant to the choice of reflections for refinement. $R$-factors based on $F^{2}$ are statistically about twice as large as those based on $F$, and $R$ - factors based on ALL data will be even larger.

Fractional atomic coordinates and isotropic or equivalent isotropic displacement parameters $\left(\AA^{2}\right)$

\begin{tabular}{lllll}
\hline & $x$ & $y$ & $z$ & $U_{\mathrm{iso}} * / U_{\mathrm{eq}}$ \\
\hline Ni1 & 1.0000 & 0.0000 & 1.5000 & $0.0107(3)$ \\
Ni2 & 0.5000 & $0.10997(4)$ & 0.5000 & $0.0105(3)$ \\
P1 & $0.68525(10)$ & 0.0000 & $1.1161(2)$ & $0.0094(3)$ \\
O1 & $0.8428(3)$ & 0.0000 & $1.1229(6)$ & $0.0127(6)$ \\
O2 & $0.6046(3)$ & 0.0000 & $0.7859(6)$ & $0.0111(6)$ \\
O3 & $0.6554(2)$ & $-0.09749(16)$ & $1.2706(4)$ & $0.0127(5)$ \\
O4 & $1.0974(2)$ & $0.11417(14)$ & $1.3104(5)$ & $0.0145(5)$ \\
H1 & 1.1208 & 0.0890 & 1.1618 & $0.022^{*}$ \\
H2 & 1.1722 & 0.1344 & 1.4293 & $0.022^{*}$ \\
O5 & $0.3964(2)$ & $0.22217(18)$ & $0.2226(5)$ & $0.0170(5)$ \\
H3 & 0.3753 & 0.2054 & 0.0398 & $0.025^{*}$ \\
H4 & 0.4440 & 0.2759 & 0.2315 & $0.025^{*}$ \\
\hline
\end{tabular}

Atomic displacement parameters $\left(\AA^{2}\right)$

\begin{tabular}{lllllll}
\hline & $U^{11}$ & $U^{22}$ & $U^{33}$ & $U^{12}$ & $U^{13}$ & $U^{23}$ \\
\hline Ni1 & $0.0091(4)$ & $0.0104(5)$ & $0.0115(4)$ & 0.000 & $0.0007(3)$ & 0.000 \\
Ni2 & $0.0103(4)$ & $0.0087(4)$ & $0.0117(4)$ & 0.000 & $0.0015(3)$ & 0.000 \\
P1 & $0.0069(6)$ & $0.0100(7)$ & $0.0105(6)$ & 0.000 & $0.0006(4)$ & 0.000 \\
O1 & $0.0069(14)$ & $0.0174(17)$ & $0.0127(14)$ & 0.000 & $0.0003(11)$ & 0.000 \\
O2 & $0.0099(14)$ & $0.0094(16)$ & $0.0127(15)$ & 0.000 & $0.0006(11)$ & 0.000 \\
O3 & $0.0129(11)$ & $0.0122(12)$ & $0.0127(11)$ & $0.0011(8)$ & $0.0026(9)$ & $-0.0004(9)$ \\
O4 & $0.0119(11)$ & $0.0171(14)$ & $0.0143(11)$ & $-0.0009(9)$ & $0.0028(9)$ & $-0.0005(9)$ \\
O5 & $0.0204(12)$ & $0.0143(12)$ & $0.0146(11)$ & $0.0017(10)$ & $0.0015(9)$ & $0.0025(10)$ \\
\hline
\end{tabular}

Geometric parameters $\left(\stackrel{\AA}{\circ}{ }^{o}\right)$

\begin{tabular}{llll}
\hline $\mathrm{Ni} 1-\mathrm{O} 1$ & $2.030(3)$ & $\mathrm{Ni} 2-\mathrm{Ni}^{\mathrm{v}}$ & $2.9086(14)$ \\
$\mathrm{Ni} 1-\mathrm{O} 1^{\mathrm{i}}$ & $2.030(3)$ & $\mathrm{P} 1-\mathrm{O} 2$ & $1.539(3)$ \\
$\mathrm{Ni1}-\mathrm{O} 4^{\mathrm{i}}$ & $2.105(2)$ & $\mathrm{P} 1-\mathrm{O} 3^{\mathrm{ii}}$ & $1.540(2)$ \\
$\mathrm{Ni} 1-\mathrm{O} 4$ & $2.105(2)$ & $\mathrm{P} 1-\mathrm{O} 3$ & $1.540(2)$ \\
$\mathrm{Ni} 1-\mathrm{O} 4^{\mathrm{ii}}$ & $2.105(2)$ & $\mathrm{P} 1-\mathrm{O} 1$ & $1.562(3)$
\end{tabular}




\begin{tabular}{|c|c|c|c|}
\hline $\mathrm{Ni} 1-\mathrm{O} 4^{\mathrm{iii}}$ & $2.105(2)$ & $\mathrm{O} 2-\mathrm{Ni} 2^{\mathrm{v}}$ & $2.063(2)$ \\
\hline $\mathrm{Ni} 2-\mathrm{O}^{\text {iv }}$ & $2.061(2)$ & $\mathrm{O} 3-\mathrm{Ni} 2^{\mathrm{vi}}$ & $2.097(2)$ \\
\hline $\mathrm{Ni} 2-\mathrm{O} 5$ & $2.061(2)$ & $\mathrm{O} 4-\mathrm{H} 1$ & 0.8500 \\
\hline $\mathrm{Ni} 2-\mathrm{O} 2^{\mathrm{v}}$ & $2.063(2)$ & $\mathrm{O} 4-\mathrm{H} 2$ & 0.8499 \\
\hline $\mathrm{Ni} 2-\mathrm{O} 2$ & $2.063(2)$ & $\mathrm{O} 5-\mathrm{H} 3$ & 0.8499 \\
\hline $\mathrm{Ni} 2-\mathrm{O} 3^{\mathrm{vi}}$ & $2.097(2)$ & $\mathrm{O} 5-\mathrm{H} 4$ & 0.8499 \\
\hline $\mathrm{Ni} 2-\mathrm{O} 3^{\mathrm{vii}}$ & $2.097(2)$ & & \\
\hline $\mathrm{O} 1-\mathrm{Ni} 1-\mathrm{O} 1^{\mathrm{i}}$ & 180.0 & $\mathrm{O} 2^{\mathrm{v}}-\mathrm{Ni} 2-\mathrm{O}^{\mathrm{vii}}$ & $86.31(10)$ \\
\hline $\mathrm{O} 1-\mathrm{Ni} 1-\mathrm{O} 4^{\mathrm{i}}$ & $91.36(8)$ & $\mathrm{O} 2-\mathrm{Ni} 2-\mathrm{O}^{\mathrm{vii}}$ & $87.33(9)$ \\
\hline $\mathrm{O} 1^{\mathrm{i}}-\mathrm{Ni} 1-\mathrm{O} 4^{\mathrm{i}}$ & $88.64(8)$ & $\mathrm{O} 3^{\mathrm{vi}-\mathrm{Ni} 2-\mathrm{O}^{\mathrm{vii}}}$ & $170.97(12)$ \\
\hline $\mathrm{O} 1-\mathrm{Ni} 1-\mathrm{O} 4$ & $88.64(8)$ & $\mathrm{O}^{\mathrm{iv}}-\mathrm{Ni} 2-\mathrm{Ni} 2^{\mathrm{v}}$ & $136.04(7)$ \\
\hline $\mathrm{O} 1$ - $-\mathrm{Ni1}-\mathrm{O} 4$ & $91.36(8)$ & $\mathrm{O} 5-\mathrm{Ni} 2-\mathrm{Ni} 2^{\mathrm{v}}$ & $136.04(7)$ \\
\hline $\mathrm{O} 4-\mathrm{Ni} 1-\mathrm{O} 4$ & 180.0 & $\mathrm{O} 2^{\mathrm{v}}-\mathrm{Ni} 2-\mathrm{Ni} 2^{\mathrm{v}}$ & $45.19(6)$ \\
\hline $\mathrm{O} 1-\mathrm{Ni} 1-\mathrm{O} 4^{\mathrm{ii}}$ & $88.64(8)$ & $\mathrm{O} 2-\mathrm{Ni} 2-\mathrm{Ni}^{\mathrm{v}}$ & $45.19(6)$ \\
\hline 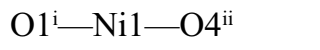 & $91.36(8)$ & $\mathrm{O}^{\mathrm{vi}}-\mathrm{Ni} 2-\mathrm{Ni} 2^{\mathrm{v}}$ & $85.49(6)$ \\
\hline $\mathrm{O} 4^{\mathrm{i}}-\mathrm{Ni} 1-\mathrm{O} 4^{\mathrm{ii}}$ & $88.32(12)$ & $\mathrm{O}^{\mathrm{vii}}-\mathrm{Ni} 2-\mathrm{Ni} 2^{\mathrm{v}}$ & $85.49(6)$ \\
\hline $\mathrm{O} 4-\mathrm{Ni} 1-\mathrm{O} 4^{\mathrm{ii}}$ & $91.68(12)$ & $\mathrm{O} 2-\mathrm{P} 1-\mathrm{O} 3^{\mathrm{ii}}$ & $110.48(10)$ \\
\hline $\mathrm{O} 1-\mathrm{Ni} 1-\mathrm{O} 4^{\mathrm{iii}}$ & $91.36(8)$ & $\mathrm{O} 2-\mathrm{P} 1-\mathrm{O} 3$ & $110.48(10)$ \\
\hline 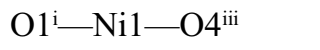 & $88.64(8)$ & $\mathrm{O} 33^{\mathrm{ii}}-\mathrm{P} 1-\mathrm{O} 3$ & $113.64(16)$ \\
\hline $\mathrm{O} 4^{\mathrm{i}}-\mathrm{Ni} 1-\mathrm{O} 4^{\mathrm{iii}}$ & $91.68(12)$ & $\mathrm{O} 2-\mathrm{P} 1-\mathrm{O} 1$ & $106.73(16)$ \\
\hline $\mathrm{O} 4-\mathrm{Ni} 1-\mathrm{O} 4^{\mathrm{iii}}$ & $88.32(12)$ & $\mathrm{O} 33^{\mathrm{ii}}-\mathrm{P} 1-\mathrm{O} 1$ & $107.60(10)$ \\
\hline 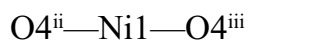 & 180.0 & $\mathrm{O} 3-\mathrm{P} 1-\mathrm{O} 1$ & $107.60(10)$ \\
\hline $\mathrm{O} 5^{\mathrm{iv}}-\mathrm{Ni} 2-\mathrm{O} 5$ & $87.92(14)$ & $\mathrm{P} 1-\mathrm{O} 1-\mathrm{Ni1}$ & $124.65(17)$ \\
\hline $\mathrm{O} 5^{\mathrm{iv}}-\mathrm{Ni} 2-\mathrm{O} 2^{\mathrm{v}}$ & $178.67(9)$ & $\mathrm{P} 1-\mathrm{O} 2-\mathrm{Ni} 2^{\mathrm{v}}$ & $133.69(7)$ \\
\hline $\mathrm{O} 5-\mathrm{Ni} 2-\mathrm{O} 2^{\mathrm{v}}$ & $90.86(9)$ & $\mathrm{P} 1-\mathrm{O} 2-\mathrm{Ni} 2$ & $133.69(7)$ \\
\hline $\mathrm{O} 5^{\mathrm{iv}}-\mathrm{Ni} 2-\mathrm{O} 2$ & $90.86(9)$ & $\mathrm{Ni} 2 \mathrm{v}-\mathrm{O} 2-\mathrm{Ni} 2$ & 89.63 (12) \\
\hline $\mathrm{O} 5-\mathrm{Ni} 2-\mathrm{O} 2$ & $178.67(9)$ & $\mathrm{P} 1-\mathrm{O} 3-\mathrm{Ni} 2^{\mathrm{vi}}$ & $123.84(12)$ \\
\hline $\mathrm{O} 2^{\mathrm{v}}-\mathrm{Ni} 2-\mathrm{O} 2$ & $90.37(12)$ & $\mathrm{Ni1}-\mathrm{O} 4-\mathrm{H} 1$ & 108.0 \\
\hline $\mathrm{O}^{\mathrm{iv}}-\mathrm{Ni} 2-\mathrm{O} 3^{\mathrm{vi}}$ & $93.26(8)$ & $\mathrm{Ni} 1-\mathrm{O} 4-\mathrm{H} 2$ & 112.1 \\
\hline $\mathrm{O} 5-\mathrm{Ni} 2-\mathrm{O}^{\mathrm{vi}}$ & $93.23(9)$ & $\mathrm{H} 1-\mathrm{O} 4-\mathrm{H} 2$ & 105.9 \\
\hline $\mathrm{O} 2^{\mathrm{v}}-\mathrm{Ni} 2-\mathrm{O}^{\mathrm{vi}}$ & $87.33(9)$ & $\mathrm{Ni} 2-\mathrm{O} 5-\mathrm{H} 3$ & 113.1 \\
\hline $\mathrm{O} 2-\mathrm{Ni} 2-\mathrm{O}^{\mathrm{vi}}$ & $86.31(10)$ & $\mathrm{Ni} 2-\mathrm{O} 5-\mathrm{H} 4$ & 112.8 \\
\hline $\mathrm{O} 5^{\mathrm{iv}}-\mathrm{Ni} 2-\mathrm{O} 3^{\mathrm{vii}}$ & $93.23(9)$ & $\mathrm{H} 3-\mathrm{O} 5-\mathrm{H} 4$ & 105.1 \\
\hline $\mathrm{O} 5-\mathrm{Ni} 2-\mathrm{O}^{\mathrm{vii}}$ & $93.26(8)$ & & \\
\hline $\mathrm{O} 2-\mathrm{P} 1-\mathrm{O} 1-\mathrm{Ni} 1$ & 180.0 & $\mathrm{O}^{\mathrm{iv}}-\mathrm{Ni} 2-\mathrm{O} 2-\mathrm{P} 1$ & $18.9(2)$ \\
\hline 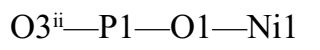 & $61.41(10)$ & $\mathrm{O} 5-\mathrm{Ni} 2-\mathrm{O} 2-\mathrm{P} 1$ & $-4(4)$ \\
\hline $\mathrm{O} 3-\mathrm{P} 1-\mathrm{O} 1-\mathrm{Ni} 1$ & $-61.41(10)$ & $\mathrm{O} 2^{\mathrm{v}}-\mathrm{Ni} 2-\mathrm{O} 2-\mathrm{P} 1$ & $-161.6(3)$ \\
\hline $\mathrm{O} 1-\mathrm{i} i 1-\mathrm{O} 1-\mathrm{P} 1$ & $-98(100)$ & 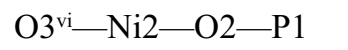 & $-74.3(2)$ \\
\hline $\mathrm{O} 4 \mathrm{i}-\mathrm{Ni1}-\mathrm{O} 1-\mathrm{P} 1$ & $45.86(6)$ & 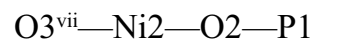 & $112.1(2)$ \\
\hline $\mathrm{O} 4-\mathrm{Ni} 1-\mathrm{O} 1-\mathrm{P} 1$ & $-134.14(6)$ & $\mathrm{Ni} 2 \mathrm{v}-\mathrm{Ni} 2-\mathrm{O} 2-\mathrm{P} 1$ & $-161.6(3)$ \\
\hline $\mathrm{O} 4{ }^{\mathrm{ii}}-\mathrm{Ni1}-\mathrm{O} 1-\mathrm{P} 1$ & $134.14(6)$ & $\mathrm{O} 5^{\mathrm{iv}}-\mathrm{Ni} 2-\mathrm{O} 2-\mathrm{Ni} 2^{\mathrm{v}}$ & $-179.48(8)$ \\
\hline 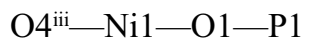 & $-45.86(6)$ & $\mathrm{O} 5-\mathrm{Ni} 2-\mathrm{O} 2-\mathrm{Ni}^{\mathrm{v}}$ & $157(4)$ \\
\hline 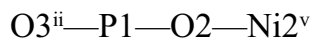 & $-140.39(17)$ & $\mathrm{O} 2^{\mathrm{v}}-\mathrm{Ni} 2-\mathrm{O} 2-\mathrm{Ni}^{\mathrm{v}}$ & 0.0 \\
\hline $\mathrm{O} 3-\mathrm{P} 1-\mathrm{O} 2-\mathrm{Ni} 2^{\mathrm{v}}$ & $-13.8(3)$ & $\mathrm{O} 3^{\mathrm{vi}}-\mathrm{Ni} 2-\mathrm{O} 2-\mathrm{Ni} 2^{\mathrm{v}}$ & $87.30(9)$ \\
\hline $\mathrm{O} 1-\mathrm{P} 1-\mathrm{O} 2-\mathrm{Ni} 2^{\mathrm{v}}$ & $102.92(19)$ & $\mathrm{O} 3^{\mathrm{vii}}-\mathrm{Ni} 2-\mathrm{O} 2-\mathrm{Ni}^{\mathrm{v}}$ & $-86.29(9)$ \\
\hline 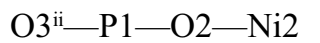 & $13.8(3)$ & $\mathrm{O} 2-\mathrm{P} 1-\mathrm{O} 3-\mathrm{Ni} 2^{\mathrm{vi}}$ & $-93.29(16)$ \\
\hline
\end{tabular}




\section{supporting information}

$\begin{array}{llll}\mathrm{O} 3-\mathrm{P} 1-\mathrm{O} 2-\mathrm{Ni} 2 & 140.39(17) & \mathrm{O} 3^{\mathrm{ii}}-\mathrm{P} 1-\mathrm{O} 3-\mathrm{Ni} 2^{\mathrm{vi}} & 31.5(2) \\ \mathrm{O} 1-\mathrm{P} 1-\mathrm{O} 2-\mathrm{Ni} 2 & -102.92(19) & \mathrm{O} 1-\mathrm{P} 1-\mathrm{O} 3-\mathrm{Ni} 2^{\mathrm{vi}} & 150.56(14)\end{array}$

Symmetry codes: (i) $-x+2,-y,-z+3$; (ii) $x,-y, z$; (iii) $-x+2, y,-z+3$; (iv) $-x+1, y,-z+1$; (v) $-x+1,-y,-z+1$; (vi) $-x+1,-y,-z+2$; (vii) $x,-y, z-1$.

Hydrogen-bond geometry $\left(\AA,{ }^{\circ}\right)$

\begin{tabular}{lllll}
\hline$D-\mathrm{H} \cdots A$ & $D-\mathrm{H}$ & $\mathrm{H} \cdots A$ & $D \cdots A$ & $D-\mathrm{H} \cdots A$ \\
\hline $\mathrm{O} 4-\mathrm{H} 1 \cdots \mathrm{O}^{\text {viii }}$ & 0.85 & 1.87 & $2.700(3)$ & 164 \\
$\mathrm{O} 4-\mathrm{H} 2 \cdots 3^{\mathrm{i}}$ & 0.85 & 1.98 & $2.731(3)$ & 147 \\
$\mathrm{O} 5-\mathrm{H} 3 \cdots 3^{\mathrm{v}}$ & 0.85 & 2.00 & $2.761(3)$ & 149 \\
$\mathrm{O} 5-\mathrm{H} 4 \cdots{ }^{\text {ix }}$ & 0.85 & 2.07 & $2.906(3)$ & 167
\end{tabular}

Symmetry codes: (i) $-x+2,-y,-z+3$; (v) $-x+1,-y,-z+1$; (viii) $-x+2,-y,-z+2$; (ix) $x-1 / 2,-y+1 / 2, z-1$. 